\title{
Rotation Transmission DEVICE IN High Ambient Hydrostatic Pressure Conditions
}

\author{
KUZMIN A., POPOV V. \& STAZHKOV S.
}

Abstract: Deep-sea mining is a significant branch of resource industry. Deep-sea drilling process and deep-sea coring are important parts of it. To improve efficiency of these two components rotation transmission device that uses the energy of hydrostatic energy was designed. Present mechanism also has an advantage of modularity, which allows to integrate it into various mining and coring systems. To simplify the mechanism and exclude pressure compensators a double sealing system is used. The first part is the gap seal, and the second part is face seals. Finding of hydrodynamic characteristics of the self-aligning mechanism that is the most significant part of the sealing are shown.

Keywords: hydrodynamics, sealing, machinery, deep-sea mining, power unit
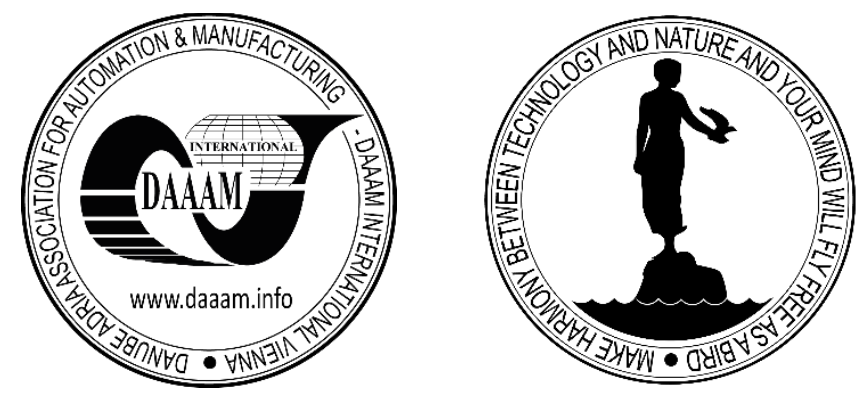

Authors' data: Kuzmin, A[nton]; Univ. Assistant Prof. Dr.techn. Popov, V[aleriy]; Univ.Prof. Dr.techn. Stazhkov, S[ergey], Batic State Technical University named after Ustinov D. F. "VOENMEH", 1-st Krasnoarmeyskaya 13, 190005, Saint-Petersburg, Russia; wutmakat@yandex.ru

This Publication has to be referred as: Kuzmin, A[nton]; Popov, V[alery] \& Stazhkov, S[ergey] (2016). Rotation Transmission Device in High Ambient Hydrostatic Pressure Conditions., Chapter 22 in DAAAM International Scientific Book 2016, pp.239-248, B. Katalinic (Ed.), Published by DAAAM International, ISBN 978-3-902734-09-9, ISSN 1726-9687, Vienna, Austria

DOI: $10.2507 /$ daaam.scibook.2016.22 
Kuzmin, A.; Popov, V. \& Stazhkov, S.: Rotation Transmission Device in High Am...

\section{Introduction}

A question of deep-sea research, sampling and mining is crucial for modern resource industry. This sphere requires proper attitude to energy, simplicity and reliability of an operating device.

In a work presented by Buckley (Buckley et al., 1994) it was shown that due to corers design it is impostant to have a power unit that provides both high torque and high speed, while having less mass as it results the whole coring process. For these causes using power unit that is based on hydrostatic pressure allows to avoid accumulators and other drawbacks of electic motors. Furthermore, no outer power supplies are needed.

Mechanism that countervail high ambient pressure at the depth of $6000 \mathrm{~m}$ was demostrated by Jia-Wang Chen (Jia-Wang Chen et al., 2013). It leads to additional mass and difficulty. In designing of deep sea equipment there is a problem of rotation into an area with high ambient pressure transmission. These conditions require secure sealing or isolation from environment and storing housing. There is a way to transmit torque using magnetic coupling (Vototyntsev B.N. et al., 2014). This method has certain restrictions. The first point is the torque can be transmitted, as there is a limit for each pair of magnets caused by a slippage. The second point is the material, as magnetic field and salt dissolved in water have noticeable effects, such as corrosion etc. The last but not the least is that such a construction has a chance to cause extensive difficulties during installation due to diameter of the coupling and the gap size. Therefore, the most reliable and simple option is using a shaft for torque transmission.

The analysis (Slyozkin N.A., 1986) showed that present structures with face seals alone of rotation transmission mechanisms do not provide high reliability at pressure values higher than $25 \mathrm{MPa}$. Herewith, these devices can be used in a narrow range of working parameters, such as pressure, rotational speed, and torque.

Therefore, it is expedient to combine a sealing unit and a bearing unit, designed to take axial shaft loads. Such a decision allows implementing this device as a single unified unit, that gives qualities of modularity to the present device, which can be integrated into more difficult systems as the piston corer (Jia-Wang Chen et al., 2013) to simplify the whole mechanism.

\section{Proposed design}

The mechanism to provide rotation transmission into environment with $60 \mathrm{MPa}$ pressure value can be designed as it is shown on figure 1 .

In the housing on the supporting bearings 2, 3 that take radial and axial load as well, the shaft 4 is mounted, which is provided with face seals 5,6 and the gap seal 7 set between them, that forms primary and secondary locking chambers with these face seals. To reduce leakage through the gap seal it is necessary to decrease its flow area and to enlarge its length. Therefore, it is rationally to perform the seal as a floating bushing, pressed to the frontal surface of the housing through the mobile spherical washer 19. The primary pressure drop is formed at the gap seal, the face seal works at 
design loads. In case of one of the seals failure, the other can withstand the full pressure drop for a certain period.

Hydrostatic pressure pushes the locking fluid from cavity 12 of the locking liquid source 10 to the primary locking chamber 8 , while the bushing 7 and the washer 19 are pressed to the face surface of the housing. The face seal 5 separates the high-pressure area and the locking fluid, and works at the pressure drop close to zero. To prevent penetration of outboard surroundings to the locking fluid, the spring 14 pressurize the locking fluid to the value that is $0,05-0,1 \mathrm{MPa}$ higher than surroundings. The bushing 7 due to its mobility along the spherical surface and mobility of the washing 19 in radial direction under hydrostatic and hydrodynamic forces is mounted coaxially to the shaft and senses its vibrations. Leakage through the gap seal get into the secondary locking chamber 9 , where the valve 15 maintains certain pressure P1, which is determined by the parameters of the face seal 6 . In this way the pressure decreases stepwise and leakages through the gap seal are reduced.

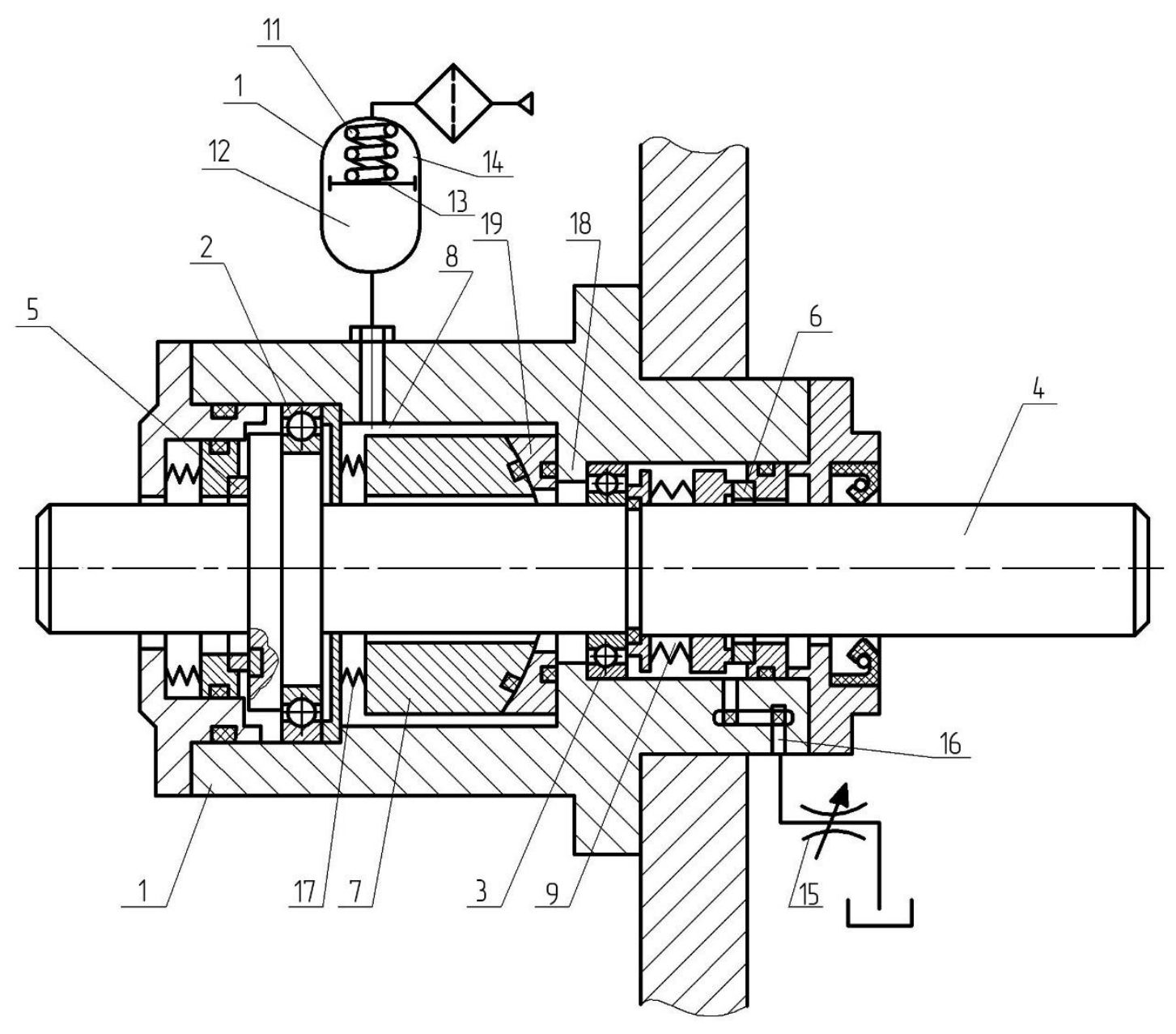

Fig. 1. The rotation transmission device design

Such a construction has a high level of reliability, as the face seal does not take the whole load of the pressure drop, and works at the certain pressure value, determined by the safety valve. In case of face seal failure, the system saves its operability. The floating bushing - spherical washing kinematic pair are forming a self-aligning 
mechanism, like piston - slipper pair in axial swash plate pumps. In contradiction to swash plate pumps, there is no such problem as constant transverse force, radial loads may take various directions, so no torque compensations or degrees of freedom restrictions (Larchikov I.A. et al., 2012) are needed.

Such constructions calculations consist in calculations of face seals, bearing unit and a gap seal, and finding of aggregate characteristics of the mechanism, such as a value of the maximum ambient pressure, permissible shaft rotational speed, leakage of the locking fluid, friction torque, working fluid temperature. Face seals and bearing unit calculation methods are well developed and accurately described (Beiselman I.D., 1975).

The chosen gap seal design caused by a combination of economic expediency and high loads. The orifice is chosen to be linear as non-linear or diaphragm type requires much accuracy and it turns out to be excessively complicated for present needs. The porous restrictors (Pascu, M, 2012) must work at high pressure, such as $60 \mathrm{MPa}$, and that aspect was not described by the authors.

Gap seal computations bring more difficulties if it is designed as a floating bushing, as it requires calculations of the pressure field in the clearance between the shaft and the bushing to define a resultant hydrodynamic force, which in necessary to find conditions of self-centering, leakages through the gap, fluid friction losses and maximum rotational speed from the dynamics point of view.

\section{Calculations}

The motion of viscous fluids is described by Navier-Stokes equations. Full mathematical conception of processes occurring in fluid must also include the continuity equation and energy equation. As a rule, for liquid motion in lubricating film Reynolds equation is used. However, every case requires confirmation of accepted assumptions. One of assumptions is permanence of locking liquid specific volume.

As a locking liquid in rotation transmission device liquids, possessing properties of lubrication, anti-corrosion, and high viscosity are used. Mineral oils meet these requirements. Pressure in present mechanism can reach up to $60 \mathrm{MPa}$. Assuming all the energy of the liquid to transit into heat, mineral oil acquires temperature of $80^{\circ} \mathrm{C}$. Mineral oil bulk modulus ranges from 1350 to $1750 \mathrm{MPa}$, thermal expansion coefficient - from $5 \cdot 10^{-4}$ to $8 \cdot 10^{-4}$. Thereby volume change of the working fluid relation of the temperature does not exceed $6,4 \%$, of the pressure $-7,4 \%$. It should be considered, that the influence of the temperature and the pressure act oppositely and partially offset each other. Therefore, it can be assumed that the working fluid is incompressible.

Most significant dependence is viscosity relation of the temperature and the pressure, while the temperature grows the viscosity increases also, while the pressure grows the viscosity reduces. As a rule research of the liquid lubrication film assume it is isothermal. That fact allows to separate hydrodynamic and heat problem. The heat problem converges to finding of the average temperature of the fluid film with the heat balance method. The fluid viscosity considered to be constant for a certain temperature. 
Navier-Stokes equations, describing the motion of the viscous incompressible fluid in operator form:

$$
\begin{aligned}
& \frac{\partial \vec{V}}{\partial t}=-(\vec{V} \cdot \nabla) \vec{V}+\vartheta \Delta \vec{V}-\frac{1}{\rho} \nabla P+\vec{F} \\
& \nabla \cdot \vec{V}=0
\end{aligned}
$$

$\nabla$ - Del operator, $\Delta$ - Laplacian, $\mathrm{t}$ - time, $\vartheta$ - kinematic viscosity coefficient, $\rho$ density, $\mathrm{P}$ - pressure, $\vec{F}$ - body forces vector field.

Assuming the fact, that the fluid motion is adiabatic, to find its temperature present equations should be used with energy equation:

$$
\Delta t=\frac{\Delta p}{\rho * c}
$$

$\Delta t$ - fluid temperature change; $\rho$ - fluid density; $\mathrm{c}$ - fluid specific heat; $\Delta p-$ pressure drop.

Present equations are true only for laminar flow. In general case stability of the laminar flow in a narrow gap with rotation (when radial clearance is rather small $\left.\frac{h}{r}<0,1\right)$ is defined by Taylor number:

$$
T_{a}=\frac{\omega * r^{\frac{1}{2}} * h^{\frac{3}{2}}}{\vartheta}
$$

$\omega$ - rotational velocity; $\quad \mathrm{r}$ - shaft radius; $\mathrm{h}$ - gap height; $\vartheta$ - kinematic viscosity coefficient. The stability condition takes a form of: $T_{a}<T_{a_{\mathrm{kp} .}}=41,3$

For rotational shaft speed range not exceeding 16 meters per second, clearance range up to 100 micrometers and locking fluid viscosity value equals $0,1 * 10^{-4} \mathrm{M}^{2} / \mathrm{c}$ Taylor number does not exceed 2,2, therefore, the stability condition is met.

The analysis of fluid motion equations system and continuity equation members importance showed relevancy of Reynolds differential equations system for fluid motion in the present design of the gap seal application.

The Reynolds equations system takes the next form:

$$
\left\{\begin{array}{c}
\frac{\partial p}{\partial x}=\mu * \frac{\partial^{2} V_{x}}{\partial y^{2}} \\
\frac{\partial p}{\partial y}=0 \\
\frac{\partial p}{\partial z}=\mu * \frac{\partial^{2} V_{z}}{\partial y^{2}} \\
\frac{\partial V_{x}}{\partial x}+\frac{\partial V_{y}}{\partial y}+\frac{\partial V_{z}}{\partial z}=0
\end{array}\right.
$$


Kuzmin, A.; Popov, V. \& Stazhkov, S.: Rotation Transmission Device in High Am...

$\mu$-dynamic viscosity coefficient.

For the present equations system solution finding it is necessary to know all the components of floating bushing and shaft surfaces points velocities that limit the fluid motion, in other words, boundary conditions.

In general the floating bushing can make spherical movements about the center of the surface of the spherical washing and plane motion with the washing perpendicularly to the shaft axis.

Supposing the shaft rotating with the frequency $\omega$. In general case the floating shaft has five degrees of freedom. To examine kinematics of the floating bushing three coordinate systems are introduced: (fig.2).

OXYZ - coordinate system with the starting point, situated on the shaft rotation axis in the center of the bushing spherical surface, $\mathrm{Z}$ axis coincides with the shaft rotation axis.

$\mathrm{O}_{1} X_{1} Y_{1} Z_{1}$ - coordinate system, related with the spherical washing, its starting point is fixed in the center of the spherical surface of the bushing. $Z_{1}$ axis is directed parallel to the shaft rotation axis.

$\mathrm{O}_{2} X_{2} Y_{2} Z_{2}$ - coordinate system, related with the floating bush, its starting point is fixed in the center of the bushing spherical surface, $Z_{2}$ axis coincides with the bushing axis.

Position of an arbitrary point $\mathrm{A}$, situated on the surface of the bushing is defined by cylindrical coordinates $\mathrm{Z}$ and $\varphi$ in $\mathrm{O}_{2} X_{2} Y_{2} Z_{2}$ coordinate system.

The floating bushing position relatively to the $O_{1} X_{1} Y_{1} Z_{1}$ coordinate system is defined by Euler angles $\alpha, \theta, \Psi$.

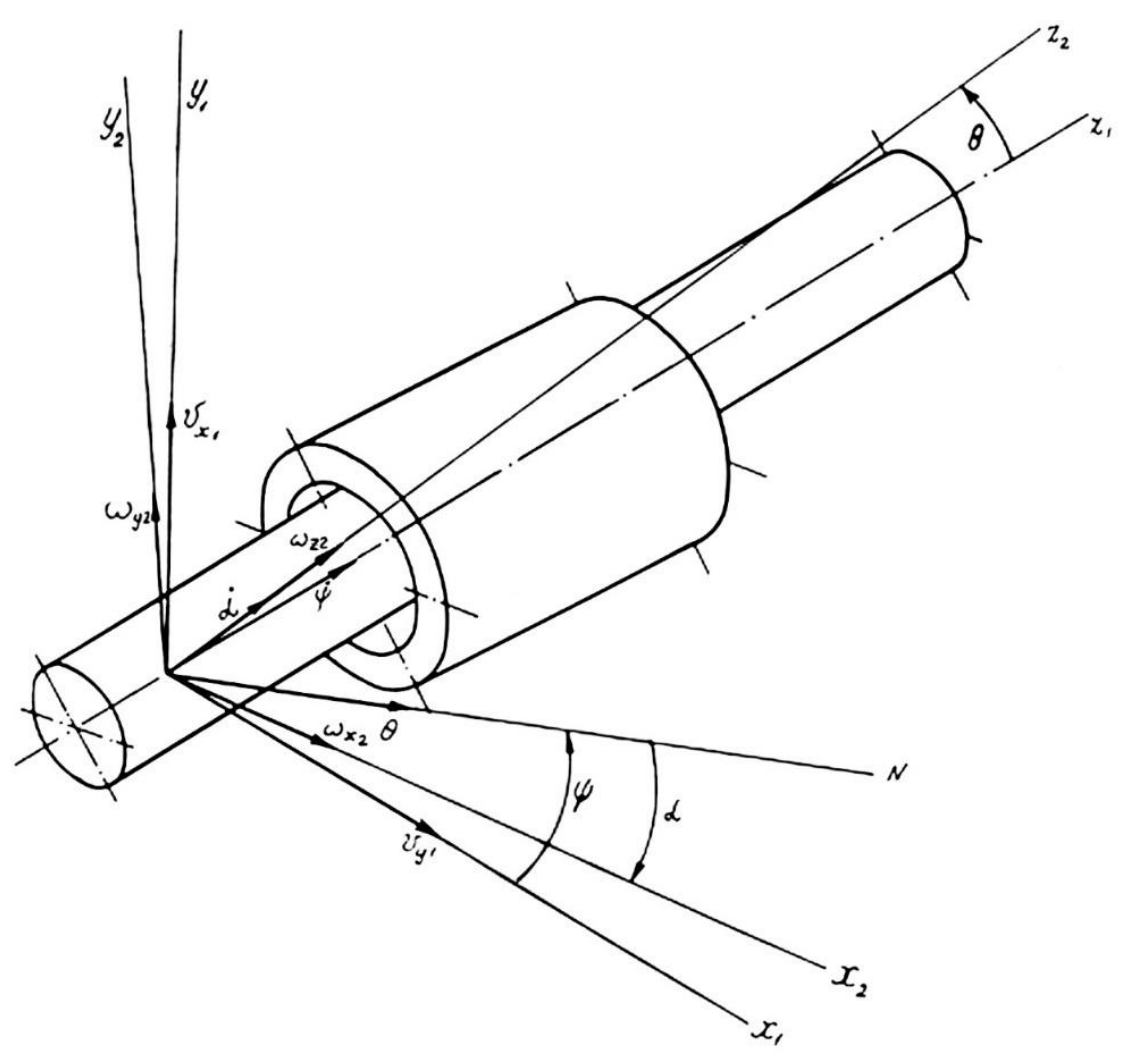

Fig. 2. Shaft-floating bushing pair 
If the floating bushing with the spherical washing makes plane movements with velocities projections $V x$ and $V y$ in $O X Y Z$ coordinate system, point A velocity projections in cylindrical coordinate system are described:

$$
\left\{\begin{array}{c}
(V p l)_{\varphi}=V_{X} * \cos \varphi-V_{y} * \sin \varphi \\
(V p l)_{r}=V_{x} \sin \varphi+V_{y} * \cos \varphi
\end{array}\right.
$$

Taking into consideration the plane motion of the bushing present equations take the next form:

$$
\left\{\begin{array}{c}
V_{r}=\left(V_{\theta}\right)_{r}+\left(V_{p l}\right)_{r} \\
V_{\varphi}=\left(V_{\theta}\right)_{\varphi}+\left(V_{\alpha}\right)_{\varphi}+\left(V_{\psi}\right)_{\varphi}+\left(V_{p l}\right)_{\varphi} \\
V_{z}=\left(V_{\theta}\right)_{z}
\end{array}\right.
$$

$\left(V_{\theta}\right)_{r, \varphi, z} ;\left(V_{\alpha}\right)_{\varphi} ;\left(V_{\psi}\right)_{\varphi}$ - point A linear velocity projections at its spherical motions on the axis of the cylindrical coordinate system, $\left(V_{p l}\right)_{r},\left(V_{p l}\right)_{\varphi}-$ point A linear velocity projections at its plane motions on the axis of the cylindrical coordinate system.

The next step is to describe the viscous incompressible fluid motion between the shaft and the floating bush. As surface radius of curvature significantly outreaches the clearance height $(\mathrm{h} / \mathrm{R}<1)$, the curvature can be neglected.

For this step a following coordinate system is needed: $\mathrm{Z}$ axis is parallel to shaft rotation axis and directed along its generatrix, curvilinear coordinate axis $\mathrm{X}$ is directed along the circumference of the shaft, $\mathrm{Y}$ axis is directed normally to the shaft surface. The starting point is situated at the intersection of the $\mathrm{Z}$ axis and the plane, passing through the center of the bushing spherical surface and perpendicular to the shaft rotation axis.

Adding boundary conditions to the Reynolds equations and conducting conversions:

$$
\begin{aligned}
& \left(h^{3} \frac{\partial P}{\partial x}\right)+\frac{\partial}{\partial z}\left(h^{3} \frac{\partial P}{\partial z}\right)=6 \mu\left[2 V_{r}+\frac{\partial h}{\partial x}\left(V_{1}-V_{\varphi}\right)-\frac{\partial h}{\partial z} * V_{z}\right] \\
& V_{1}=\omega \cdot R_{\mathrm{B}}, R_{\mathrm{B}}-\text { shaft radius. }
\end{aligned}
$$

\section{Results}

Pressure field between the floating bushing and the shaft calculations results are the basis for findings of hydrodynamics forces and torques, acting on the floating bushing, and also for power losses in gap seal findings, which consist of volume loss (locking fluid leakage) and mechanic loss (caused by friction in the fluid layer between the shaft and the bushing). 
Kuzmin, A.; Popov, V. \& Stazhkov, S.: Rotation Transmission Device in High Am...

The influence of gap seal parameters is essential information for rotation transmission device design. Certain characteristics calculations are shown on figures below.
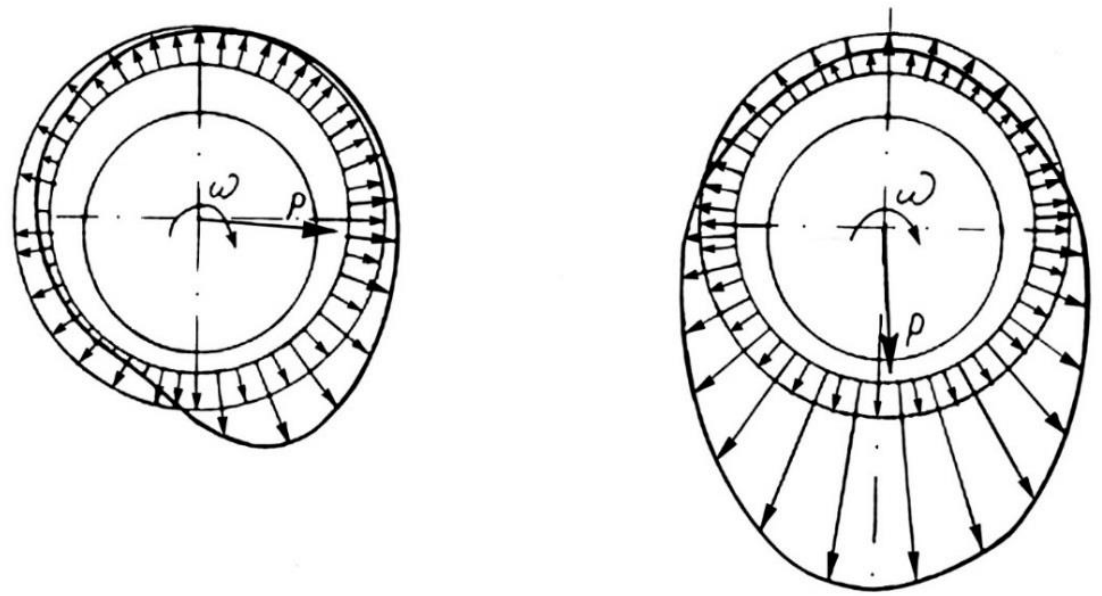

1

Fig. 3. - Coordinate X pressure distribution $(0 \mathrm{rad} / \mathrm{s}-\mathrm{left} ; 0.08 \mathrm{rad} / \mathrm{s}-\mathrm{right})$

$\mathrm{Q}, \mathrm{sm}^{\wedge} 3 / \mathrm{min}$

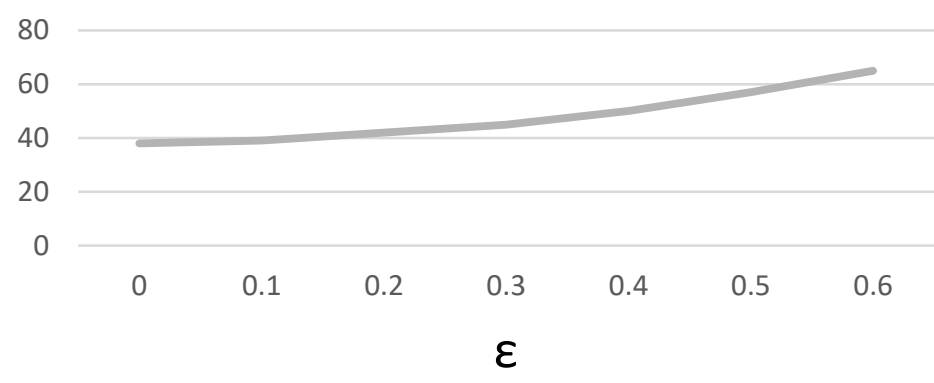

Fig. 4. The gap sealing parameters from eccentricity relationship

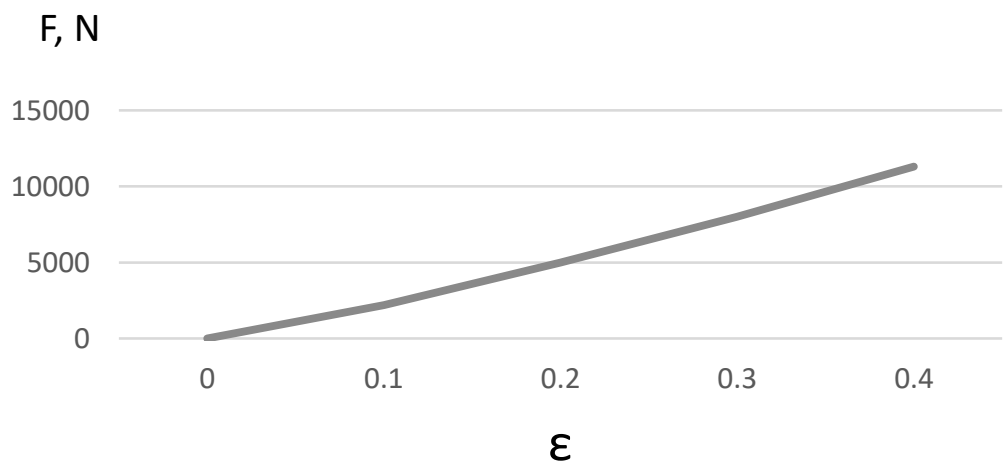

Fig. 5. The gap sealing parameters from eccentricity relationship 


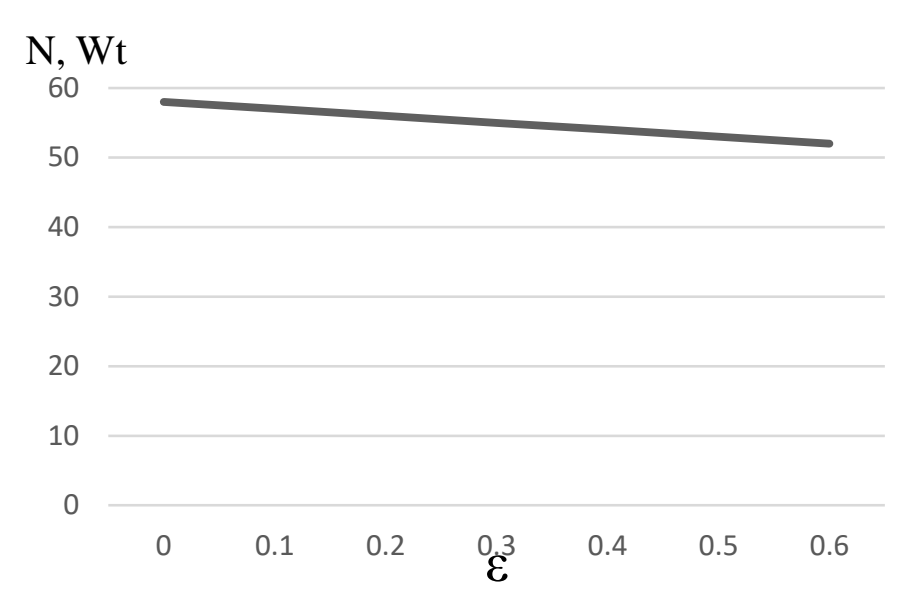

Fig. 6. The gap sealing parameters from eccentricity relationship

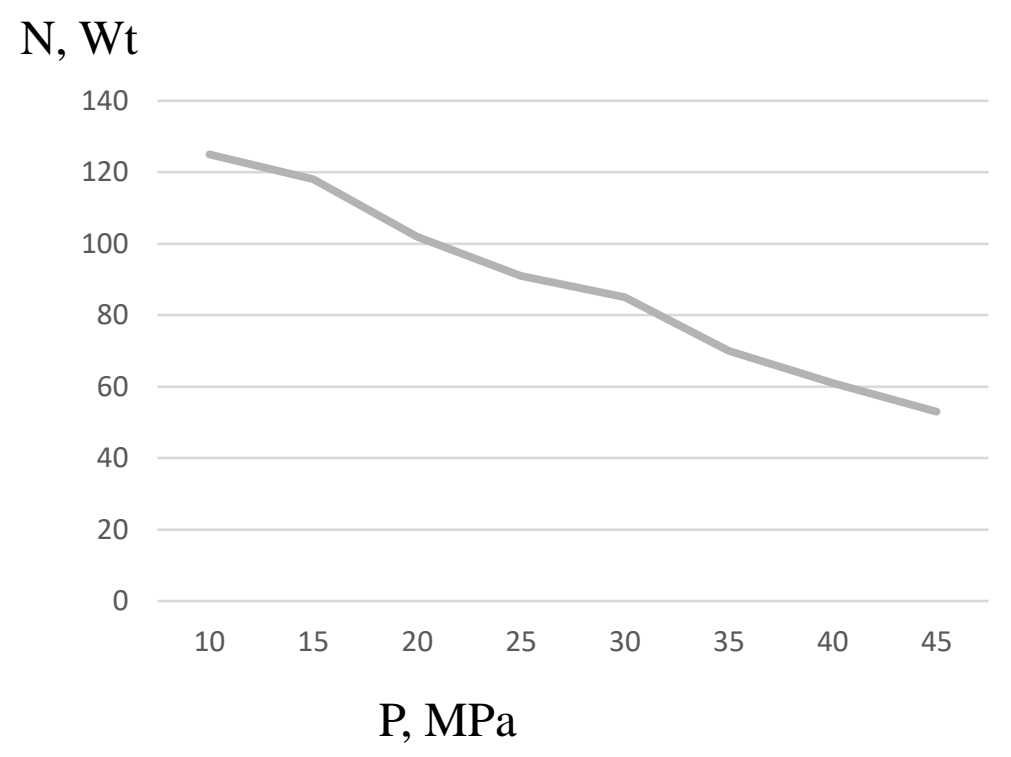

Fig. 7. The gap sealing parameters from pressure relationship

$\mathrm{N}$ - power losses, $\mathrm{F}$ - resultant pressure force, $\mathrm{Q}$ - locking fluid leakage, $\mathrm{p}$ pressure, $\varepsilon$ - relative eccentricity.

As the calculation showed, pressure growth causes significant increase of the volume losses. A decrease in centering hydrodynamic force and power losses can be explained by reducing viscosity of the locking fluid, caused by the temperature growth.

The relative eccentricity of the floating bushing relatively to the shaft has the decisive influence on the hydrodynamic force value. With eccentricity growth from 0,1 to 0,4 the force increases 4,4 times, while the leakages grow 1,25 times and power losses change insignificantly. Angular displacement of the floating bushing have influence only on hydrodynamic force value.

With increase of the shaft rotational speed hydrodynamic force and power losses are rising, while volume losses remain the same. This fact differs from information obtained by various authors for floating rings and can be explained by distinction in hydrodynamic processes in short and long gaps. 
Kuzmin, A.; Popov, V. \& Stazhkov, S.: Rotation Transmission Device in High Am...

\section{Conclusions.}

The rotation transmission device was designed. The device uses the hydrostatic pressure energy, which helps to decrease the mass of the mechanism and exclude outer power supply.

The device is assembled as one single unit, can be applied for different mechanisms.

A highly reliable deep-water rotation transmission device design was demonstrated. The hydromechanics calculations and its results, such as pressure field, were obtained. Crucial characteristics and relations were constructed. Further work includes theoretical and experimental approval of present results.

Present device allows to significantly improve deep-sea mining and coring process. Energy saving as well as possibility for new design projects to include this device as a single unit are the main results of the work.

\section{References}

Buckley D.E., MacKinnon W.G., Cranston R.E., Christian H.A (1993) Problems with piston core sampling: Mechanical and geochemical diagnosis, Available from: http:// https://www.researchgate.net/publication/240420107 Accessed: 2016-10-05

Jia-Wang Chen, Wei Fan, Brian Bingham, Ying Chen, Lin-Yi Gu and Shi-Lun li (2013) A Long Gravity-Piston Corer Developed for Seafloor Gas Hydrate Coring Utilizing an In Situ Pressure-Retained Method, Energies 2013, 6, 3353-3372, ISSN 1996-1073

Vototyntsev B.N., Osipov V.I., Stazhkov S.M., Tsvetkov V.A. (2014) Rotation Sealed Lead-In Unit of Submersible Mechanism, 25th DAAAM International Symposium on Intelligent Manufacturing and Automation, Procedia Engineering 100 ( 2015 ), pp. $1450-1454$

Slyozkin N.A. (1986) Sealing and compaction equipment, Mashinostroeniye, pp. 464, USSR

Larchikov, I. A., Stazhkov, S. M., Yurov A. V., (2012) The Study Of Hydromechanical Processes In Hydromachines Of Power-Intensive Drives, Annals of DAAAM for 2012 \& Proceedings of the 23rd International DAAAM Symposium, Volume 23, No.1, ISSN 2304-1382 ISBN 978-3-901509-91-9

Beiselman I.D., Zipkin B.V. et al. (1975) Rolling-element bearing, Mashinostroeniye, pp. 572, USSR

Pascu, M; Obrea, C. F.;Andioaia, D. \& Funaru, M.(2012) Studies And Researches Concerning The Use Of Porous Restrictor In The Case Of Hydrostatic Guideways. Annals of DAAAM for 2012 \& Proceedings of the 23rd International DAAAM Symposium, Volume 23, No.1, ISSN 2304-1382 ISBN 978-3-901509-91-9 